\title{
Review of: "The predicting factors for indication of surgery in patients with hemoperitoneum caused by corpus luteum cyst rupture"
}

Frederic Blavier

Potential competing interests: The author(s) declared that no potential competing interests exist.

This article is well written and the objective, methods, results and conclusion of the study is interesting but some major and minor improvements should be made, ideally in abstract (or methods and results) and in discussion.

Major concerns:

1. In the abstract, the authors quoted "a retrospective review of medical records of 155 patients diagnosed with hemoperitoneum caused by corpus luteum cyst rupture" but, in the Methods and Results, "92 patients/women diagnosed with hemoperitoneum with a/caused by CLCR".

2. In Methods of Methods, Corpus luteum cysts were confirmed pathologically in surgery. It should be discussed because I am not sure that it is always (or even often) needed. It is potentially damaging the ovary. It is not a best practice mostly for women with pregnancy wish but also for the risk of shortening time before menopause.

3. The authors should discuss this study of 106 women diagnosed with ruptured corpus luteal cyst with hemoperitoneum, analysing CT findings (included cyst size, cyst shape, sentinel clot sign, ring of fire sign, hemoperitoneum depth, active bleeding in portal phase and attenuation of hemoperitoneum) to determine if they can predict surgical interventions, “Lee, M. S., Moon, M. H., Woo, H., Sung, C. K., Jeon, H. W., \& Lee, T. S. (2017). Ruptured Corpus Luteal Cyst: Prediction of Clinical Outcomes with CT. Korean journal of radiology, 18(4), 607-614. https://doi.org/10.3348/kjr.2017.18.4.607". The last sentence of discussion may be changed...

Minor concerns:

1. The urine hCG test was quoted in methods without any blood hCG and without discussing the falsenegative urine hCG previously reported, for example: “Kleinschmidt, S., Dugas, J. N., Nelson, K. P., \& Feldman, J. A. (2021). False negative point-of-care urine pregnancy tests in an urban academic emergency department: a retrospective cohort study. Journal of the American College of Emergency Physicians open, 2(3), e12427. https://doi.org/10.1002/emp2.12427".

2. In Methods of Methods, I do not understand the sentence "Enhanced abdomen-pelvic CT was performed to ... locate other possible surgical abdomens". 
3. In results, it seems contradictory to quote "Initial vital signs showed no differences but pulse rate was noted higher in the surgery group". Pulse rate is not a vital sign (as quoted in Methods of Methods)? 